\title{
Lessons Learned During Three Decades of Operations of Two Prospective Bioresources
}

\author{
William E. Grizzle, Katherine C. Sexton, Diane McGarvey, ${ }^{2}$ Zachery V. Menchhofen, ${ }^{2}$ and Virginia LiVolsi ${ }^{2}$
}

Prospective collection is a model through which biospecimens are provided for research. Using this model, biospecimens are collected based on real-time requests from the research community instead of being collected based on the prediction of future requests. We describe the lessons learned by two bioresources that have operated successfully using a prospective model for over three decades. Our goal is to improve other bioresources by increasing utilization of biospecimens that honor consented donors who provide biospecimens to the research community; this provides strong evidence of stewardship of the public trust. The operation of these sites requires flexibility, close communication, and cooperation with the investigator in developing a standard operating procedure (protocol) based on the investigator's needs described in their initial request. If practicable, almost any preparation can be provided, including fresh (nonfrozen) biospecimens and tissue blots. A quality management system includes rigorous quality control of the specific biospecimens provided to an investigator. The informatics approach focuses on the investigator, the investigator's request, and the biospecimens collected for the investigator; the informatics focus of classic biobanks is on the biospecimens collected to match expected future requests. These lessons have been incorporated into our current operations. Standard investigator agreements (e.g., indemnification and no unapproved biospecimen transfers to third parties) replace material transfer agreements. We have operated under the prospective model of the Cooperative Human Tissue Network (CHTN), which has been successful and has provided over 1.2 million biospecimens since it began in 1987. These tissues have supported over 4300 peer-reviewed scientific articles. Since 2012, about 1000 publications have indicated support by CHTN tissues; their average citation rate is 31 with an $\mathrm{H}$ factor of 61 . Also, during this period, 114 patents cited the CHTN. We also describe disadvantages of prospective bioresources (e.g., inadequate distribution of rare tissues, biospecimens not immediately available, and delayed clinical outcomes).

Keywords: biospecimen, prospective model, biobank, bioresource, biodistributor, utilization, human tissues, publications, patents, citations, $\mathrm{H}$ factor

\section{Introduction}

$\mathrm{P}$ ROSPECTIVE COLLECTION AND DISTRIBUTION is one of several models used to provide biospecimens to support biomedical and other research. ${ }^{1-3}$ A prospective bioresource is a bioresource model, in which biospecimens are collected after an investigator specifically requests them to support an active or future research project; thus, the prospective bioresource is investigator centric. In contrast, a "classic biobank" is biospecimen centric and collects biospecimens based on the expectation of future investigator requests. The classic biobank usually collects remnant clinical specimens from surgery, autopsy and/or cytology, and may collect from deceased organ donors and consented living organ donors.
Similarly, the prospective bioresource collects investigator requested biospecimens from these same sources. Thus, the difference is what the investigator wants versus what biospecimens are available.

This article describes the lessons learned by two prospective bioresources that have operated successfully using a prospective model for over three decades. The two prospective biorepositories are located at the University of Alabama at Birmingham (UAB), Birmingham, AL, and the University of Pennsylvania (UPENN), Philadelphia, PA. Both prospective bioresources have been members of the Cooperative Human Tissue Network (CHTN) since its establishment in 1987, and both utilize the same prospective model used by the CHTN. UAB also has operated several

\footnotetext{
${ }^{1}$ Division of Anatomic Pathology, Department of Pathology, The University of Alabama at Birmingham, Birmingham, Alabama.

${ }^{2}$ Department of Pathology, University of Pennsylvania, Philadelphia, Pennsylvania.
} 
classic biobanks and adds these experiences and observations to this article. The focus of this article is to aid in establishing and improving other bioresources by increasing utilization rates of biospecimens. Increased utilization honors donors who have consented to provide biospecimens to the research community and, hence, provides strong evidence of stewardship of the public trust.

In the prospective model, investigators request biospecimens based on specifying the detailed characteristics of the human tissue biospecimens needed to support their research. This requires a specific diagnosis of the tissues needed, not just vague descriptions such as "breast cancers," which could include a broad range of breast cancers such as sarcomas of the breast. A more specific diagnosis, for example, would be $\mathrm{ER}^{+}$/ $\mathrm{PR}^{+}$ductal adenocarcinoma of the breast. Donor features (e.g., race) may be specified along with other requirements of the biospecimens such as a request for fresh solid tissues with matching paraffin blocks as well as numbers and sizes of biospecimens, for example, 40 biospecimens of $250 \mu \mathrm{L}$ of EDTA plasma from patients with lung adenocarcinoma. Also, tissue processing (e.g., fresh [not frozen] samples of a solid cancer), storage, and shipping requirements should be described.

These characteristics are included in a detailed application. Upon receipt, the application is carefully reviewed by senior bioresource personnel and by a pathologist who may be the medical director of the bioresource or the bioresource principal investigator. Issues such as practicality and specificity of the requests are carefully considered and problematic issues are resolved among senior bioresource personnel and the investigator. Because the collection of biospecimens is closely associated with the investigator request, the prospective bioresource is strongly linked to specimen distribution to investigators.

For each investigator request, a standard operating procedure (SOP) or protocol is developed via close interactions between the investigator and the bioresource. In contrast, a banking model typically has only a few SOPs that specify how biospecimens of the biobank are collected, processed, and stored, and these may vary with the biospecimen type. Hence, the biospecimens in a classic biobank are relatively uniform, and investigators are offered biospecimens that are readily available, usually through a catalog mechanism. Prospective collection versus biobanking can be described from the investigator's view as specifying defined specimen characteristics followed by prospective specimen collection, processing, short-term storage, and rapid distribution versus distribution of previously collected biospecimens from the inventory of a biobank.

This prospective model has led to highly focused efforts in collecting and distributing biospecimens to investigators. The success of this model is demonstrated by the following statistics of the CHTN. As of December 2017, investigators had been provided with about 1.2 million biospecimens that have supported over 4300 articles in peer-reviewed journals. From 2012 through February 2018, CHTN biospecimens supported $\sim 1000$ published peer-reviewed articles; the average citation rate of these articles is 31 , and the $\mathrm{H}$ factor is 61 . Also, during this period, 114 issued patents cited the CHTN.

Over the three decades of their operation, UAB and UPENN have identified many important lessons in the operation of prospective bioresources. This article describes lessons learned during this period. We suggest that these aspects of operation be incorporated into prospective bior- esources that are being planned, are in operation, or even into the operations of classic biobanks.

\section{Advantages and Disadvantages of Prospective Collection Compared to General Banking}

In this article, we try to avoid the terms "biobank, biovault, biorepository, etc." because the connotations of those terms are that biospecimens are collected and locked away until potential future use which may not occur. ${ }^{1-6}$ We prefer the term "biodistributor," which focuses on what should be the goal of all biobanks - distribution and/or use of biospecimens in research. Alternatively, we use "bioresource" as in this article. Contrasts between a prospective bioresource and classic biobanks are as follows:

- A prospective bioresource is efficient in that most of its collected biospecimens are distributed to investigators. This is a financial advantage based on cost recovery and avoids the ethical dilemma of collecting biospecimens from consented donors and not using these biospecimens in research. It also minimizes even the concept of biohoarding. ${ }^{6}$

- The prospective bioresource provides a tailored product to an investigator. This product may be more useful to meet the investigator's specific research requirements than biospecimens collected under a few standard SOPs.

- Prospective bioresources frequently provide fresh (nonfrozen) biospecimens, thus avoiding possible artifacts (e.g., molecular changes; modified proteomic signatures) that may occur or be introduced by freezing or storage. There is little to no understanding of such potential artifacts. $^{7-9}$

- Biospecimens prospectively collected for investigators are more likely to be used rapidly and hence may minimize molecular changes that may occur during long-term storage. $^{7-9}$

- Prospective bioresources have the disadvantage that requested biospecimens usually will not be immediately available because they must be collected. This is especially a limitation for providing biospecimens of rare diseases/conditions that cannot be readily collected by one institution, even within several years.

- A related disadvantage of prospective collection at some sites is the potential for relatively slow procurement of various types of biospecimens in addition to rare biospecimens. The time required to fulfill investigators' requests can be exacerbated by complex investigator requirements. This may be accentuated by surgery and surgical pathology requirements/protocols, tissue factors, and current approaches to medical care such as neoadjuvant therapy. ${ }^{7-11}$ Determining feasibility and timeframes are based, in part, both on the experience of the site pathologist and other personnel as well as the capability of the informatics system to identify factors that may delay distribution of specific types of biospecimens. Of note, a model to estimate the future needs for frozen tissues by biorepositories based on similar factors as well as research use factors, biomedical funding factors, research trends and biobank trends has been published. ${ }^{10}$ Such a modified model could be of use to aid prospective bioresources in planning distribution.

- A major disadvantage of prospective collection is that clinical outcomes are not rapidly available to investigators 
because biospecimens must "mature" before a clinical outcome is reached. This period of maturation is relatively short for very aggressive cancers such as pancreatic ductal adenocarcinomas (e.g., $<4$ years), but longer ( $>4$ years) for tumors such as colorectal and mammary cancers.

- The disadvantages of prospective collection are the advantages of a classic biobank. For example, the classic biobank may permit selected biospecimens to mature; this approach provides investigators the opportunity to utilize multiple criteria in preselection of biospecimens and, hence, aid in selecting larger and stronger cohorts for their studies. ${ }^{3} \mathrm{Al}-$ though we frequently criticize utilization of biospecimens from classic biobanks and other bioresources, the importance of biobanks in biomedical research is huge. For example, a study of Canadian researchers who were funded by the Cancer Research Society (of Canada) over 2010-2014 found that $38 \%$ of their publications used biospecimens. Of these, $36 \%$ of biospecimens were obtained from biorepositories, most of which were classic biobanks. ${ }^{11}$

UAB has operated both prospective bioresources as well as classic biobanks under the umbrella of the UAB Tissue Collection and Banking Facility (TCBF). The tissues collected for the prospective bioresources at $\mathrm{UAB}$ are essentially all used if collections meet the investigator requests as to size, quality control (QC), and other investigator-defined characteristics; however, all biospecimen factors may not be known when tissues are first collected. Of importance, some UAB biorepositories are "closed" and may only be used by a defined group of users via a Tissue Utilization Committee (TUC). Our experience is that utilization of biospecimens of such biorepositories frequently is less than 20\%; however, we have found that a TUC usually will agree to provide requested biospecimens from the closed collection to junior investigators at participating sites and to other investigators with extramural peer-reviewed funding.

When a UAB biobank has lost funding, assuming the consent was broad enough to allow other research uses, biospecimens from the biobank have been offered to investigators associated with our prospective bioresources. If the inventory of the biobank is large and many of its biospecimens do not meet current investigator requests (e.g., these biospecimens usually are from limited diagnostic groups), it may take longer than 5 years to distribute the inventory of the biobank. Of importance, we have found that other UAB closed biobanks, not under TCBF control, also are sometimes willing to provide specimens to investigators associated with our prospective bioresources; this has been an important source of biospecimens for less common cancers (e.g., brain).

\section{Practical Lessons Learned as to Operations of a Prospective Bioresource}

The following are some of the practical lessons we have learned based on our operation of prospective bioresources as well as via other biobanking models. In many cases, the lessons can be adapted to any bioresource; however, the prospective bioresource has all of its operation and cost recovery based on targeting specific biospecimens requested by investigators and the types of surgery that may yield these requested biospecimens. The cost recovery for this model is based on the responsibility of the requesting investigator to pay for the defined cost recovery charge for each specimen collected to meet the defined characteristics of the specified request. Thus, we have emphasized the lessons that tend be more important to prospective bioresources even though they may be also important, but to a lesser extent, to other bioresource models.

- Although all biorepositories must have Institutional Review Board (IRB)/Ethics approval, the application for IRB/Ethics approval for a prospective bioresource is somewhat different from a classic biobank. The prospective model utilizes an open demographic subject enrollment from whom biospecimens are collected; thus, the demographics are determined by the requests of the investigators. We require investigators to have local IRB/ Ethics review/approval, but most of the investigators of an open prospective bioresource may not be local. Thus, IRB/Ethics reviews/approvals are from many different IRBs/Ethics groups. Our prospective bioresources also prefer to use a Limited Data Set requiring investigators to sign a Data Use Agreement (DUA) so that elements of dates that investigators may request can be provided. Also, dates may be useful to a prospective bioresource for biospecimen labeling. In addition, the DUA protects data associated with biospecimens and ensures that no attempts are made to identify donors.

- The application that investigators must submit to request biospecimens from a prospective bioresource are critical to its operation. A standard application is required, which, via answers to detailed questions, provides specific and extensive descriptions of the biospecimens needed by the investigator. Because collections and cost recovery are based on this description, this application typically must be more detailed than one that is used by a classic biobank that ultimately provides biospecimens that are available from its inventory.

- There should be careful initial review of the submitted application documents to ensure practicality and specificity of requests. Clear, direct communication between senior bioresource personnel and the investigator are necessary to resolve all questions and other issues before acceptance of the application.

- Initial decisions must be made by bioresource leadership as to which requests for biospecimens will not be accepted (e.g., biospecimens requiring too much effort, tissues infected with bloodborne pathogens, tissues requiring research activities such as the determination of a cancer's molecular phenotype). These parameters are discussed with investigators so that they understand the limitation of the prospective bioresource, especially that the prospective bioresource will not perform research for the investigator.

- Acceptable specimen characteristics should be defined and codified for the bioresource; for example, the CHTN defines an acceptable cancer specimen as one containing at least $10 \%$ cancer. $^{12}$ This prevents confusion as to charges for cost recovery.

- The application establishes essentially a contract between the prospective bioresource and the investigator and institution. The application should include protection of the bioresource by providing a clear agreement between the prospective bioresource and each investigator/institution, including indemnifications, requirements for biosafety education, recognition in publications, and cost recovery. 
Prohibitions include transfer of biospecimens to third parties without approval, attempts to identify donors, and specific commercialization practices (e.g., marketing of biospecimens or their components to third parties).

- The mechanism for cost recovery of the prospective bioresource should be defined to aid in financial stability and to demonstrate responsiveness to stakeholders. Because collections of specific biospecimens are expensive and some biospecimens collected following specific investigator requirements may not be useful to other investigators, it is recommended that as soon as a biospecimen meeting investigator requirements is collected and its diagnosis confirmed, the requesting investigator becomes responsible for its cost.

- To cover shipping costs, each investigator should have a shipping account with a designated courier. It is also critical that the investigator provide to the bioresource a valid shipping address. It is important that this information be identified before specimen collection, as a prospective bioresource that is rapidly distributing specimens does not have the time or resources to attempt to define these issues at the time of collection. A surcharge by the bioresource to cover added supplies (e.g., shipping boxes) should be considered.

- Because of the high daily volume of shipping by an open prospective bioresource and the need to ship biospecimens rapidly without delay, several employees trained in the requirements/regulations of the International Air Transport Association are needed.

- A focus by the prospective bioresource on rapid distribution of biospecimens to investigators has the advantage of minimizing molecular changes and storage requirements, and it facilitates cost recovery.

- Because prospective collection requires carefully and clearly defined biospecimen diagnoses and other characteristics, the clinical formalin-fixed paraffin-embedded (FFPE) block archives of pathology can be searched for cases that may meet the needs of investigators requesting slides and/or cores for constructing tissue arrays. This approach depends on clearly defined agreements with components of anatomic pathology and the capabilities of the informatics systems that support such searches.

- Prospective bioresources need a robust, flexible informatics approach that primarily focuses on the investigator, prospective biospecimen collection, and biospecimen distribution.

- A nonredundant informatics vocabulary, which identifies many biospecimen characteristics, especially biospecimen diagnosis and donor characteristics, is important to a prospective bioresource. This vocabulary ensures that requested biospecimen characteristics are uniquely defined and minimizes the collection of biospecimens that are inappropriate for specific investigator requests.

- Close collaboration in a network with other like-minded open prospective bioresources to improve the distribution of consistently described, collected, processed, and distributed biospecimens is important. Such collaboration can foster development of a common procedure manual and business rules. Sharing of prospective requests makes the operations of all prospective bioresources more efficient.

- The use of a robust quality management system (QMS) with rigorous QC of collected biospecimens is necessary.
For prospective collection, it is critical to perform QC when biospecimens are collected to confirm that they meet investigator requests. ${ }^{1,2,12-16} \mathrm{SOP} /$ protocols with each investigator should be developed for their requests and, hence, the SOPs tend to be investigator-centric.

- It is important for the prospective bioresource to follow the trends in research. Because the prospective bioresource depends on rapidly establishing agreements with investigators as to the biospecimens investigators currently need, it is especially important for the prospective bioresource to rapidly identify trends in research. This facilitates preparing the prospective bioresource for meeting new types of biospecimen requests. For example, increasing use by investigators of fresh biospecimens and FFPE blocks and slides have required bioresource planning for different approaches to distribution and seeking of new sources of biospecimens. Our prospective bioresources had to change their approaches and applications based on these changes.

- SOPs also should be developed for all bioresource activities, including biospecimen collection, processing, storage, QC, and distribution. ${ }^{1,2,12,14-16}$

- A well-defined process of consenting donors for the use of their biospecimens in research is necessary. In the United States, when the new Common Rule regulations go into effect, newly collected biospecimens that are identified in the bioresource and may be used for unspecified research may require a Broad consent, even if the specimens are distributed as de-identified. ${ }^{17-19}$

- All applications from investigators to a bioresource must be accompanied by evidence of review by the investigator's IRB/Ethics Board.

- Participation in a professional organization of other bioresources such as the ISBER (International Society for Biological and Environmental Repositories) provides opportunities for the sharing of experiences and gaining of knowledge. ${ }^{15,16}$

- Detailed training (e.g., safety, regulatory, and ethical) and rapid performance evaluation of personnel is needed. In our experience, performance issues not associated with training frequently do not improve after a probationary period. Thus, it is important not to maintain inadequately functioning personnel, as they are a danger to a bioresource and its employees.

- Ongoing evaluation of service and quality, including annual surveys of investigators, should be performed. ${ }^{1,2,13-16}$

- Providing investigators with an opportunity to provide rapid feedback regarding each shipment is necessary to quickly identify problems with bioresource operations. ${ }^{1,2,13-16}$

- It is important to address problems identified by investigators in a timely manner.

- A marketing approach is needed to make potential users aware of the bioresource and thus ensure a wide range of users resulting in efficient operations.

- A decision to provide biospecimens to for-profit companies is beneficial to the advancement of medicine.

- Tracking and documentation of usage information, including publications demonstrating the benefits and successes of the bioresource is very useful. This should include a description of the impact of these successes on medical care and may require the part-time services of a medical editor. 


\section{Investigator Interactions in Defining Their Prospective Protocols}

When investigators request tissues and/or data to support their research, many are uncertain as to their exact needs for specific tissues as well as the potential characteristics of the requested tissue. In addition, some investigators do not understand the morphology, molecular features, and biology of specific tissues. It is critical that the characteristics of requested biospecimens be distinctly defined by investigators based on their research project ${ }^{1-3}$; however, investigators may require some help from the bioresource in establishing the specific collection protocol.

Specifically, if a protocol were developed for an investigator to obtain "lung cancer," it would be too vague and might lead to problems when such biospecimens are distributed, especially because some of the biospecimens collected under vague descriptions ultimately may not meet the investigator's needs. For a "lung cancer" protocol, a bioresource might collect a wide range of lung cancers such as neuroendocrine carcinoma; however, the investigator may actually only want a specific subtype of lung cancer such as adenocarcinoma of the lung, and even the request for these tumors might be limited to those with a specific molecular subtype (e.g., anaplastic lymphoma kinase (ALK) mutations).

Of note, investigators requesting molecular features not characteristically documented as part of standards of clinical care may have to accept biospecimens for which they have to screen for the molecular markers as part of their research. Thus, for adequate prospective collections to be efficient, investigators must provide or be prompted by the prospective bioresource to provide specifics as to exactly what types of biospecimens are acceptable. Similar problems would arise if donor characteristics were not clearly specified; for example, the investigator may ask for breast cancers from young women without specifying an age range. Because investigators' perceptions of "young" may differ from those of the prospective bioresource, the investigators should be prompted to specify an age range that is acceptable to them. In some cases, it may be more important for a protocol to specify reproductive status (e.g., pre- or postmenopausal), which may vary with different age ranges.

Most assays used in research may require only a relatively small biosample of a tumor such as $0.1 \mathrm{~g}$, yet, an investigator may request $5 \mathrm{~g}$ of the tumor just to ensure that there is enough for future projects. This is problematic in that some tumors are relatively small and not a large amount of tumor will remain after diagnostic examination. Thus, investigators must understand that requesting $5 \mathrm{~g}$ of some tumors will greatly reduce the availability of biospecimens that can fulfill the investigator's needs. It is, therefore, critical that the bioresource make the investigator aware of such limitations, and encourage a reduction of size requirements whenever possible to increase the feasibility of prospective collection. Also, if $5 \mathrm{~g}$ is required, multiple requests from different investigators requiring smaller samples should be filled before the larger request. Of importance, the professional bioresource personnel may assist the investigator in suggesting modifications to study techniques, allowing the usage of smaller samples.

When multiple requirements are made as to the characteristics of biospecimens, the difficulty of obtaining such biospecimens may increase exponentially. For example, if 10,
$0.1 \mathrm{~g}$ biospecimens of ductal adenocarcinoma of the breast are requested, the protocol is not difficult. In contrast, if 10, $0.1 \mathrm{~g}$ specimens of triple-negative ductal adenocarcinoma of the breast (about $20 \%$ of breast cancers) are needed from chemotherapy/radiation naive women who are between 30 and 40 years of age, to be shipped fresh in media, the protocol quickly becomes difficult to fulfill. In these situations, it is essential that bioresource personnel communicate with the investigator about the feasibility of sample collection based on their current request. In some cases, the very low probability of successful collection should prevent the acceptance of the request by the bioresource.

\section{Types of Specimens Distributed to Investigators}

Prospective bioresources provide many different preparations of tissues, depending on requests of investigators. Specifically, plasma/buffy coats based on multiple different anticoagulants (e.g., EDTA, heparin, or citrate) could be provided instead of one type of plasma. Similarly, while most paraffin blocks and fixed tissues are based on fixation in $10 \%$ neutral buffered formalin, both paraffin blocks and fixed tissues may be prepared using a wide range of other fixatives; these could include zinc formalin, acid formalin, alcoholic formalin, Bouin's, 70\% ethanol, and high heat.

Other preparations could include frozen or fresh samples of blood components (e.g., serum), urine, and saliva; fresh solid tissues, frozen or fixed solid tissues, FFPE blocks, paraffin embedded blocks with tissue fixed by alternative methods, tissue microarrays, frozen sections, paraffinunstained tissue sections, hematoxylin and eosin-stained sections, sections stained by other techniques, and tissue blots (e.g., on nitrocellulose). RNA and DNA preparations can be extracted from diseased tissues, but there have not been many requests for these products because investigators typically want to control the extraction methods used in their research. Of note, RNA previously banked by some biorepositories proved problematic when microRNAs were not present in the RNAs extracted by previous methods. The previous methods did not retain small RNA fragments, which were thought to be degraded RNA.

A prospective bioresource can allow investigators to develop complex collection protocols consisting of multiple preservation methods (e.g., a fresh sample in specific media along with a matched frozen sample and a Bouin's fixed paraffin block). Specimens with complex processing are unlikely to be available from classic biobanks. Thus, investigators might be relegated to accepting suboptimal samples from biobanks based on availability and might have to modify their study approaches accordingly.

In the last decade, there have been greatly increased requests for FFPE material because RNA and DNA can now be analyzed in FFPE sections (e.g., in situ hybridization). ${ }^{20}$ The FFPE aliquots and/or frozen sections also can be macrodissected before analysis. In addition, prospective bioresources have observed an increasing trend of requests for fresh tissues due to new technologies such as those focusing on research based on analysis of single cells. Of note, most classic biorepositories may not be able to provide large numbers of fresh specimens.

In general, a prospective service has to be flexible and responsive to providing different types of samples requested by investigators. The prospective bioresource should, if requested, provide annotations as to the samples provided. 
The standard information provided with each specimen by our prospective bioresources includes the patient's age, sex, and race, the QC diagnosis of the specific specimen aliquot provided a de-identified copy of the surgical pathology report with documented patient diagnosis, and, for cancer specimens, information as to whether or not the patient received neoadjuvant therapy. The surgical pathology report for most cancers provides the grade and pathologic stage of the cancer.

Other information from the electronic medical record and/or charts such as a patient history of systemic disease, for example, type 1 diabetes mellitus or a family history of ovarian cancer and smoking history can be collected for an investigator, but collection of such additional information should result in added charges. All national and regional regulations regarding donor privacy should be observed throughout the entire workflow, especially in the distribution of samples and associated donor information.

\section{Limitations to Provision of Tissues to Investigators}

Decisions have to be made as to what tissues and services will or will not be provided and to whom. For example, will specimens be provided to researchers at for-profit/ commercial companies? It is our view that many medical advances are made by commercial companies and that some medical breakthroughs, especially the development of medical approaches/drugs, would not be possible without the involvement of for-profit organizations. Therefore, it is our opinion that it is important to provide for-profit companies with specimens for their research as well as for product development. However, any investigators, including commercial entities, receiving tissues must agree not to further market the tissues (e.g., to sell aliquots or molecular extracts from biospecimens). In addition, the potential for provision of biospecimens to for-profit companies should be indicated in the informed consent.

Although we obtain tissues to meet prospective requests from classic and other biobanks, it is our policy not to provide tissue to investigators or organizations for the establishment of biobanks or to allow investigators to build up their existing tissue banks. Providing such tissue to banks would result in loss of control of these specimens as to prospective agreements, may introduce artifacts, and may prevent their timely use in research.

Processing should not include performing some of the investigators' research or separating cells from tissue or constructing organoids. Any prospective bioresource also may decide not to collect specific specimens. For example, the CHTN does not collect fresh specimens from donors with a history of bloodborne pathogens (e.g., hepatitis C), and fetal tissues are not provided. ${ }^{12}$ Importantly, some tissue requests may require too much effort/resources. For example, UAB has refused to collect whole vertebral columns from autopsies due to the extensive effort required. Of critical importance, diagnosis can never be compromised by obtaining specimens for research nor can autopsy patients be disfigured (e.g., eyes removed) without special consent.

\section{Priority of Distribution}

Investigators requesting prospective tissues should be assigned a priority for tissue collection and assignment. We utilize a rotating priority such that an investigator who receives a specimen then goes to the bottom of the request list for that specific specimen type. Some modifications to priority can be made for special situations such as when an investigator needs only two or three specimens to complete an article.

\section{Marketing}

Our prospective bioresources operate primarily in the United States and Canada with international requests considered on a per case basis. Frequently, prospective bioresources depend upon requests from investigators outside local institutions; this requires a wide range of investigators to be aware of prospective bioresource services. Of importance, investigators should know that a prospective bioresource will adjust collection, processing, temporary storage, and distribution to meet their exact needs rather than providing "standard specimens."

Marketing has always been a challenge to our prospective bioresources, and we still find that many investigators who need human tissues are unaware of our existence and/or services. Most investigators contact us because of recommendations from their colleagues. Besides these word-ofmouth contacts, we try to inform investigators of our services through individual websites as well as the website of the CHTN. The CHTN also maintains an active social media presence.

The CHTN has developed a brochure that is distributed along with branded promotional materials from exhibit booths at the annual meetings of the American Association for Cancer Research (AACR) and the Federation of American Societies for Experimental Biology (FASEB). Booths also are used at some topical meetings sponsored by the AACR. Our personnel are available at these and other meetings to help investigators in developing their prospective protocols. Over the years, we as members of the CHTN have attended other annual meetings, including the American Society of Clinical Oncology and the Endocrine Society as members, presenters, and exhibitors. Evaluation of investigator contacts at these meetings did not warrant further attendance; however, choice of meetings to attend as an exhibitor depends upon the tissue focus of the bioresource.

The CHTN also uses periodic mailings to explain prospective collection services to investigators who have grants from the National Cancer Institute (NCI). In general, marketing continues to be one of the major challenges of our prospective biorepositories.

\section{Quality Management System}

All biorepositories, whether prospective or not, need a rigorous QMS. The importance and features of a QMS have been described previously. ${ }^{1,2,13-16}$ As part of the QMS, there should be QC of all bioresource products and output. Because of space limitations in this article, we have elected to highlight only the QC of solid tissues; this QC is critical to the operation of prospective bioresources.

In general, the diagnosis of a patient described in the surgical pathology report does not adequately describe the characteristics of biospecimens actually provided to an investigator. Thus, a bioresource should perform QC on 
an aliquot representative of each solid tissue specimen provided. $^{1,2,13-16}$ The minimum QC is based on the actual specimen provided to an investigator and is described on the CHTN website (https://www.chtn.org). Typically, a central aliquot is collected from a tissue and the aliquot is used to construct an FFPE block. A minimum QC is a microscopic examination of stained sections by a board-certified pathologist. For cancers, QC may include the $\%$ tumor in the specimen and of the tumor, the $\%$ fibrosis, $\%$ mucin, and $\%$ necrosis as well as identification of the $\%$ malignant cells (sometimes designated \% tumor nuclei). For example, if there are $40 \%$ lymphocytes and plasma cells and minimal other nonmalignant cells mixed with the malignant cells of a tumor, the $\%$ of malignant cells is about 60\% (https:// www.chtn.org).

The QC needs to be performed quickly to facilitate the rapid distribution of prospective tissues. Nevertheless, for fresh tissues, the investigator may need to accept the tissue before QC is available. If QC is not performed until biospecimens are distributed, as occurs in some classic biobanks, then the biobank actually is storing some unusable samples, which is a waste of resources. Also, the biobank is never certain as to its actual biospecimen inventory; this may lead to promising biospecimens that are unavailable and/or inappropriate to investigators for their needs.

\section{Defining an Acceptable Specimen for Distribution}

Thus, it is our recommendation that bioresources in general, when a biospecimen is prospectively collected for an investigator, the characteristics of the biospecimen are unknown at collection, yet, the bioresource incurs a cost. This can range between $\$ 100$ and $\$ 300$ based on CHTN cost calculations. Prospectively collecting, processing, storing, and distributing biospecimens are expensive, so minimum requirements for an acceptable biospecimen should be specified. For example, the CHTN sets the minimum amount of tumor for a malignant specimen to be acceptable as $10 \%$ of the specimen. ${ }^{12}$ If the investigator's study requires a higher amount of tumor, this can be established and included in the development of the protocol, but may result in additional charges.

When biospecimens are prospectively collected for an investigator and they meet the minimum standard, the CHTN requires that the investigator accept/pay a cost recovery fee for this specimen (see subsequent discussion in Costs to Investigator section). Such fees are necessary to keep the investigators from changing their requirements after specimens have been collected, and from abandoning areas of research without compensating the prospective bioresource for the efforts expended to collect specimens for the investigator.

\section{Agreements with Investigators}

For prospective and other bioresources, it is important to develop written agreements between the bioresource and the investigator requesting biospecimens and the investigator's institution/company. For the CHTN, these agreements serve as material transfer agreements. For a prospective bior- esource, it is important that the investigator agrees to accept all specimens collected, which meet the specifications of their established protocol. The CHTN requires the investigators to educate personnel in biohazards of tissue and associated safety measures to indemnify the provider of the tissue samples, to recognize the CHTN in all publications resulting, in part, from the specimens provided, and not to attempt to identify donors of the tissues. The investigator must also agree not to transfer specimens to third parties without the approval of the CHTN.

The reason for the requirement for indemnification is that bioresources do not want to be legally responsible for injuries that might be caused by the tissues they provide. Potential infections by various potential organisms following cuts and other injuries due to specimen handling become the responsibility of the recipient organization. Similarly, agreements for specimens transferred to third parties may not be valid unless the third party approves and also signs an agreement. Recognition of the provision of tissues by bioresources in publications is very important in that it is an index of the usefulness of the bioresource. The recognition can be in the acknowledgments section of the publication, but some investigators choose to recognize the CHTN in the methods section. Suggested wording for such acknowledgments can be included in agreements to ensure that this recognition is enforced.

\section{Costs to Investigators}

It is our view that investigators place more value and respect on biospecimens for which processing fees are associated than for biospecimens provided free of charge; thus, it is our strong recommendation that processing fees be assessed to investigators for services provided by the bioresource. In our experience the cost of prospective collection of one specimen ranges from $\$ 100$ to $\$ 300$ based on an economy of scale; if only one specimen is collected and distributed in a year, the cost of that specimen is the total cost of the bioresource operation for that year. It is very important to periodically monitor the costs for collecting, processing, performing QC, temporarily storing, and distributing a specimen. For the bioresource to be financially viable, all costs must be recovered. Without some external financial support, all costs would have to be transferred to the investigator.

For some bioresources, institutional and philanthropic support and/or grants cover some of the costs, so only a portion of the actual specimen costs are charged to the investigator. Even when there is bioresource support, all costs of specimen collection should be charged to for-profit/ commercial companies. If this concept is not followed, the costs to academic and other nonprofit institutions actually are, in part, subsidizing for-profit companies. The cost recovery charges to investigators are essential to supplement or cover the budget of the prospective bioresource and to cover unexpected costs such as equipment repair/replacement. Few biorepositories can be self-sustaining.

The CHTN is funded by the NCI and, as such, has determined that cost recovery charges to academic investigators should be maintained as low as possible, while for-profit commercial companies are invoiced charges for full specimen cost recovery. 


\section{A Partial Banking Component for Prospective Biorepositories}

Even a prospective bioresource is likely to have a limited banking operation. Specifically, when a specimen of tumor is being prospectively collected, there may be excess tumor and related tissues available that are likely to be used in the future. Most biorepositories will collect, process, and bank the additional tissues for future use. It is the experience of the CHTN that many investigators may want to use these banked tissues, even though they may not exactly meet their current tissue requirements. Also, rare tissues may be identified, for which there are no current protocols; however, except for rare specimens, the primary focus of this bioresource should be on the relatively shortterm distribution of biospecimens via this banking component. The addition of a biobanking component is not a merger of the prospective model with a classic biobank. Specifically, there is no attempt by the biobank to collect a wide range of biospecimens, rather the biobanking component has a very specific focus on biospecimens, for which there is evidence of potential utilization in the immediate future. Also, none of the advantages of the classic biobank is maintained, such as focus on biospecimen maturity.

\section{Informatics of Prospective Biorepositories}

The design of an informatics software application and database for use in a prospective bioresource must take into account and track several operational factors and data elements not found in other informatic approaches of classic biobank models. There may be similarities between the informatics system of a classic biobank and a prospective bioresource, such as the collection of data metric annotations, specimen collection tracking data, storage and inventory data, and quality management annotations; however, the focus of the prospective bioresources' informatics approaches must be investigator-centric.

To be responsive to the broad range of potential requests that can occur with a prospective procurement model, the structure of an informatics program must be able to query multiple data sources to provide accurate metrics regarding the feasibility of obtaining specific sample types. This may include access to the institution's clinical databases as well as historical and/or archival data of the bioresource. It is critical to consider that the retrieved data must be analyzed and interpreted by a management team that is abreast of current clinical techniques that can affect (positively and negatively) the availability of biospecimens for research purposes.

The informatics approach of the prospective bioresource focuses primarily on tracking individual investigators, investigators' protocols, and robust distribution and utilization metrics for biospecimens assigned to each investigator. In contrast to the classic biobank model, in which the focus is on the annotation of the stored specimens and their disposition, an investigator-centric application system must have the ability to annotate data under multiple protocols of one investigator. This is because one investigator frequently may have collection protocols for multiple different requests (e.g., one for prostate, another for breast).
The vocabulary and data structure must allow for any variant of data annotation to be collected within the application system. This vocabulary must be nonredundant in both its structure and underlying values. This allows for the greatest latitude of future reporting, searching the collected data, and permits matching investigator's protocols with the diagnosis of specimens programmatically as specimens become available. While the use of free-type fields is often not advisable in a prospective procurement model, a data application system needs to have the flexibility to describe all aspects of an investigator's protocol. This can be achieved by the judicial use of a limited number of freetype fields.

The architecture of the data system must be flexible enough to allow for the addition of future data elements and/or integration with future modules or data systems. There also should be clear contact information so that an investigator or designees can be reliably and rapidly contacted when specimens become available. Sample matching or data search functions should be easily assessable so that when an aliquot of lung adenocarcinoma becomes available, personnel can quickly try to match the specimen with those investigators requesting comparable types of lung tissues. In contrast, the informatics program for a model based purely on biobanking will be primarily focused on the available tissues in the biobank and will be less focused on an investigator's needs because available specimens usually cannot be exactly matched to an investigator's protocol.

\section{Evaluation of the Success of a Prospective Bioresource}

Evaluating a prospective bioresource requires investigator feedback. Whether or not investigators have received biospecimens from the bioresource, all investigators whose requests for tissues have been accepted as reasonable protocols should be asked periodically to evaluate the bioresource. In the case of a classic biobank, only those investigators who receive specimens typically are asked to evaluate the biobank. Therefore, the approach to evaluating a prospective bioresource differs from other models of biobanking.

Bioresource evaluations may be general (e.g., annual request for feedback) and/or shipment focused (e.g., quality of samples within a specific shipment). The use of evaluations and the bioresource response to the evaluation feedback should be incorporated into the bioresource QMS.

Besides investigator feedback, there are multiple other measures of the success of a prospective bioresource. These include biospecimen distribution to investigators that should be separated into various categories to identify trends (e.g., preservation, anatomic sites, diagnoses). Publications and patents supported by distributed biospecimens should be tracked. The impact factor of journals where articles are published and the citations of the articles as well as scientific and medical advances associated with the reported discoveries should be noted. Although investigators who utilize prospective bioresources are asked to acknowledge the contributions of the bioresource in their publications and to inform the bioresource of their publications, this is frequently not done. To judge and emphasize the importance of publications, we have found it very useful to utilize a 
medical editor to aid in identifying relevant publications and in evaluating their impact. The medical editor can be aided by the pathologists supporting the prospective bioresources in identifying the medical importance and impact of related publications.

\section{Arrangements with Different Sites}

It has been the experience of the CHTN that a subcontract or fee-for-service arrangement with other institutions may be useful to assist in meeting some investigator protocols. Thus, the cooperating site collects specimens under the well-defined SOPs of the primary site and provides specimens to the primary site for distribution to investigators. Of note, such arrangements may be best funded by a fee-for-service arrangement (i.e., the cooperating site is only paid based on the needed specimens they actually provide and which are distributed to investigators).

\section{Summary}

Because the prospective bioresource has the primary goal of distributing high-quality biospecimens to investigators, including those at for-profit companies, this model has proved to be very efficient and cost effective for supporting biomedical research of high impact. The prospective bioresource must have great flexibility to meet a wide range of varying requests for specifically collected and processed biospecimens. This model requires close interaction between investigators and the prospective bioresources with each investigator request being developed into a practical and specific protocol that meets the investigator's actual needs for biospecimens. The diagnosis of each biospecimen is supported by a QMS with rigorous and rapid QC. The operation of the prospective bioresource should be user friendly with a view that each investigator's project deserves great attention without needless bureaucracy. Investigator agreements protect the bioresources and assure appropriate biospecimen utilization. An informatics approach also is necessary that is investigator-centric and different from the biospecimen-centric model utilized in classic biobanking.

The prospective bioresource model may be more labor intensive than a classic model, but subsequent high utilization rates demonstrate its value as a dynamic asset to the research community. Equally important is that the rapid distribution and use of biosamples ensures that the intentions of the specimen donors have been honored.

The tissues collected for the prospective bioresources at UAB and UPENN are essentially all used if they meet the investigator requests as to size, QC, and other investigatordefined characteristics.

\section{Acknowledgments}

Supported by CHTN grants 1UM1CA183728 to UAB and 5UM1CA183711 to UPENN; the Tissue Procurement Shared Facility of the UAB Comprehensive Cancer Center P30CA13148-41; the Pulmonary Hypertension Breakthrough Initiative 1R24HL123767-01; and the Hepato/Renal Fibrocystic Diseases Core Center P30DK074038. Presented, in part, at the 2018 Annual Meeting of the International Society for Biological and Environmental Repositories (ISBER).

\section{Author Disclosure Statement}

No conflicting financial interests exist.

\section{References}

1. Grizzle WE, Bell WC, Sexton KC. Issues in collecting, processing and storing human tissues and associated information to support biomedical research. In: Srivastava S, Grizzle WE (eds). Translational Pathology of Early Cancer. Amsterdam, The Netherlands: IOS Press BV; 2011: 531-549.

2. Grizzle WE, Sexton KC, Bell WC. Issues in operating a human tissue resource supporting biomedical research. In: Areman E, Loper K (eds). Cellular Therapy: Principles, Methods and Regulations, 2nd edn. Bethesda, MD: AABB; 2016:497-509.

3. Grizzle WE, Bledsoe MJ, Al Diffalha S, Otali D, Sexton KC. The utilization of biospecimens: Impact of the choice of biobanking model. Biopreserv Biobank (In press).

4. Hewitt R. Why all the names? Trans-Hit BiomarkersImproving ethical access to biosamples for research. March 27, 2018. Available at: https://www.linkedin.com/pulse/ biobank-biorepository-biolibrary-biovault-biohoard-biotrusthewitt (accessed September 4, 2018).

5. iSpecimen, Inc. A Worldwide Study of the Factors Affecting Sustainable Biobanking Operations and TechnologyBased Approaches to Increase Utilization Rates: An Independent Survey. Lexington, MA: iSpecimen, Inc.; 2018.

6. Catchpoole D. 'Biohoarding': Treasures not seen, stories not told. J Health Serv Res Policy 2015. DOI: 10.1177/ 1355819615599014.

7. Otali D, Al Diffalha S, Grizzle WE. Biological, Medical, and other Tissue Variables Affecting Biospecimen Utilization. Biopreserv Biobank (In press).

8. Atherton DS, Sexton KC, Otali D, Bell WC, Grizzle WE. Factors affecting the use of human tissues in biomedical research: Implications in the design and operation of a biorepository In: Grützmann R, Pilarsky C (eds). Cancer Gene Profiling: Methods and Protocols, 2nd edn. New York: Springer Science+Business Media, LLC; 2016: $1-38$.

9. Grizzle WE, Otali D, Sexton KC, Atherton DS. Effects of cold ischemia on gene expression: A review and commentary. Biopreserv Biobank 2016;14:548-558.

10. Meredith AJ, Slotty A, Matzie LK, et al. A model to estimate frozen tissue collection targets in biobanks to support cancer research. Biopreserv Biobank 2015;13: 356-362.

11. Castillo-Pelayo T, Babinszky S, LeBlanc J, et al. The importance of biobanking in cancer research. Biopreserv Biobank 2015;12:172-177.

12. Cooperative Human Tissue Network: CHTN. Available at: https://www.chtn.org/faqs.html (accessed September 7, 2018).

13. Grizzle WE, Sexton KC, Bell WC. Quality assurance in tissue resources supporting biomedical research. Cell Preserv Technol 2008;6:113-118. 
14. Grizzle WE, Gunter EW, Sexton KC, Bell WC. Quality management of biorepositories. Biopreserv Biobank 2015; 13:183-194.

15. Campbell LD, Betsou F, Garcia DL, et al. 2012 Best practices for repositories: Collection, storage, retrieval and distribution of biological materials for research. Biopreserv Biobank 2012;10:79-161.

16. Astrin JJ, Baker S, Barker KJ, et al. Best Practices: Recommendations for Repositories. 4 ed. Vancouver: ISBER; 2018:1-104.

17. Bledsoe MJ, Russell-Einhorn MK, Grizzle WE. Shifting sands: The complexities and uncertainties of the evolving US regulatory, policy, and scientific landscape for biospecimen research. Diagn Histopathol 2018;24: DOI: 10.1016/ j.mpdhp.2017.09.004.

18. Health and Human Services, Office for Human Research Protections. Attachment A-FAQs Relating to Recommendations on Broad Consent, 2018. Available at: https:// www.hhs.gov/ohrp/sachrp-committee/recommendations/ attachment-a-faqs-relating-to-recommendations-on-broadconsent/index.html (accessed September 6, 2018).

19. Health and Human Services, Office for Human Research Protections. Attachment C-Updated FAQs on Informed
Consent for Use of Biospecimens and Data, 2018. Available at: https://www.hhs.gov/ohrp/sachrp-committee/ recommendations/attachment-c-faqs-recommendations-andglossary-informed-consent-and-research-use-of-biospecimensand-associated-data/index.html (accessed September 6, 2018).

20. Gaffney EF, Riegman PH, Grizzle WE, Watson PH. Factors that drive the increasing use of FFPE tissue in basic and translational cancer research. Biotech Histochem 2018; DOI: $10.1080 / 10520295.2018 .1446101$.

Address correspondence to: William E. Grizzle, MD, PhD Division of Anatomic Pathology

Department of Pathology The University of Alabama at Birmingham

Zeigler Research Building

Room 408

703 19th Street South Birmingham, AL 35294-0007

E-mail: wgrizzle@uabmc.edu 